OPEN ACCESS

Edited by:

Michael S. Okun,

University of Florida Health,

United States

Reviewed by:

Philip Starr,

University of California, San

Francisco, United States

Tipu Z. Aziz,

John Radcliffe Hospital,

United Kingdom

*Correspondence:

Helen M. Bronte-Stewart

hbs@stanford.edu

Specialty section:

This article was submitted to Brain Imaging and Stimulation,

a section of the journal

Frontiers in Human Neuroscience

Received: 30 June 2020 Accepted: 05 August 2020 Published: 31 August 2020

Citation:

Bronte-Stewart HM, Petrucci MN,

O'Day JJ, Afzal MF, Parker JE,

Kehnemouyi YM, Wilkins KB,

Orthlieb GC and Hoffman SL

(2020) Perspective: Evolution of Control Variables and Policies for Closed-Loop Deep Brain Stimulation

for Parkinson's Disease

Using Bidirectional

Deep-Brain-Computer Interfaces.

Front. Hum. Neurosci. 14:353.

doi: 10.3389/fnhum.2020.00353

\section{Perspective: Evolution of Control Variables and Policies for Closed-Loop Deep Brain Stimulation for Parkinson's Disease Using Bidirectional Deep-Brain-Computer Interfaces}

\author{
Helen M. Bronte-Stewart ${ }^{1,2 *}$, Matthew N. Petrucci ${ }^{1}$, Johanna J. O'Day ${ }^{1,3}$, \\ Muhammad Furqan Afzal ${ }^{1,4}$, Jordan E. Parker ${ }^{1}$, Yasmine M. Kehnemouyi ${ }^{1}$, \\ Kevin B. Wilkins ${ }^{1}$, Gerrit C. Orthlieb ${ }^{1}$ and Shannon L. Hoffman ${ }^{1}$
}

${ }^{1}$ Department of Neurology and Neurological Sciences, Stanford University School of Medicine, Stanford, CA, United States, ${ }^{2}$ Department of Neurosurgery, Stanford University School of Medicine, Stanford, CA, United States, ${ }^{3}$ Department of Bioengineering, Stanford University, Stanford, CA, United States, ${ }^{4}$ Graduate School of Biomedical Sciences, Icahn School of Medicine at Mount Sinai, New York, NY, United States

A deep brain stimulation system capable of closed-loop neuromodulation is a type of bidirectional deep brain-computer interface $(\mathrm{dBCl})$, in which neural signals are recorded, decoded, and then used as the input commands for neuromodulation at the same site in the brain. The challenge in assuring successful implementation of bidirectional $\mathrm{dBCls}$ in Parkinson's disease (PD) is to discover and decode stable, robust and reliable neural inputs that can be tracked during stimulation, and to optimize neurostimulation patterns and parameters (control policies) for motor behaviors at the brain interface, which are customized to the individual. In this perspective, we will outline the work done in our lab regarding the evolution of the discovery of neural and behavioral control variables relevant to PD, the development of a novel personalized dual-threshold control policy relevant to the individual's therapeutic window and the application of these to investigations of closed-loop STN DBS driven by neural or kinematic inputs, using the first generation of bidirectional $\mathrm{dBCls.}$

Keywords: brain-computer interface $(\mathrm{BCl})$, beta oscillations, Parkinson's disease, deep brain stimulation, subthalamic nucleus, closed-loop neurostimulation, kinematics, brain-machine interface (BMI)

\section{INTRODUCTION}

Continuous deep brain stimulation (DBS) is an established therapy for cardinal motor signs in Parkinson's disease (PD; Krack et al., 2003; Deuschl et al., 2006; Schuepbach et al., 2013; Edwards et al., 2017). Current DBS systems operate in an open-loop manner: the neurostimulator cannot sense the neural signals from the brain that interfaces with the deep brain electrode(s), and which it is modulating. It applies a continuous regular train of electrical pulses of fixed frequency, amplitude, and pulse width, which cannot automatically adjust to different symptoms, the individual's state of activity or medication cycle. These limitations may contribute to dyskinesias and speech, 
mood, and cognitive impairments (Weaver et al., 2005; Deuschl et al., 2006; Williams et al., 2010). A major unmet need in neuromodulation for neuropsychiatric diseases is the development of a closed-loop neurostimulator: a bidirectional deep brain-computer interface $(\mathrm{dBCI})$, in which neural signals are recorded, decoded and then used as the input commands for neuromodulation at the same site in the brain (Mussa-Ivaldi et al., 2010; Fetz, 2015).

One challenge for bidirectional dBCIs in movement disorders is the need to discover neural inputs relevant to pathological motor behaviors. Such neural inputs need to be robust, reliable, specific to the individual and their activity state, and which can be recorded during stimulation. Unlike traditional BCIs where the normal neural code is used to restore function, the neural signals available from DBS leads in PD represent pathological neural code; the desired neural activity has to be extrapolated from animals or simulations (Wichmann et al., 1994; Nini et al., 1995; He, 2014; Feingold et al., 2015). Another challenge for bidirectional dBCIs is to discover control policies (patterns and parameters of neurostimulation) that will optimize specific motor behaviors.

In this perspective, we will outline the work done in our lab regarding the evolution of the discovery of neural and behavioral control variables relevant to PD, the development of a novel personalized dual-threshold control policy relevant to the individual's therapeutic window and the application of these to investigations of closed-loop STN DBS driven by neural or kinematic inputs, using the first generation of bidirectional dBCIs.

\section{Deconstructing the Resting-State Neural Code Relevant to Parkinson's Disease}

Exaggerated neuronal oscillations and synchrony in alpha and beta frequencies $(8-30 \mathrm{~Hz})$ have been demonstrated in the sensorimotor network during the resting state in $\mathrm{PD}$, which can be termed the resting state beta oscillopathy (Bergman et al., 1994; Nini et al., 1995; Bevan et al., 2002; Levy et al., 2002; Brown, 2003; Schnitzler and Gross, 2005). Many early reports were limited to one short neural recording and it was questioned whether the beta oscillopathy was a stable feature across individuals (Priori et al., 2013). Our intra-operative recordings demonstrated that the resting state beta oscillopathy was stationary, in that it re-occurred, unchanged, over time despite intervening periods of movement or neurostimulation (Bronte-Stewart et al., 2009). In freely moving individuals with $\mathrm{PD}$, the resting state beta spectral profile was conserved in different resting postures (Quinn et al., 2015). It was also similar and coherent between the STNs of an individual, although different among individuals, and was coherent with the motor cortex-STN hyper direct pathway efferent projection sites, suggesting that it is a property of the widespread sensorimotor network (de Solages et al., 2010; Whitmer et al., 2012). A computerized peak detection algorithm confirmed the presence of a resting state beta oscillopathy in 129 out of 130 STNs (Shreve et al., 2017).

Initially, it was debated whether the beta oscillopathy was an epiphenomenon or linked to Parkinson's disease pathophysiology. Evidence suggesting that it is related to progressive pathophysiology was supported by the demonstration that it only emerged several days after inducing Parkinsonism in rodents and was not evident after acute blockade of dopamine receptors (Mallet et al., 2008), and from neural recordings in the non-human primate model of progressive Parkinsonism (Dorval et al., 2015; Muralidharan et al., 2016; Wang et al., 2017). Intra-operative bilateral STN neural recordings (112 STNs) in individuals with PD demonstrated that there was significantly greater resting-state beta band power in the more affected STN when compared to the lesser affected STN, further suggesting a relationship to disease progression (Shreve et al., 2017). As part of a longitudinal study, the resting state beta band power increased over time in the untreated STN, in two individuals with PD, who had bilateral STN DBS leads placed but who chose to have only one side activated (Trager et al., 2016).

The demonstration that the STN beta oscillopathy was attenuated by therapeutic doses of dopaminergic medication and intensities of STN DBS established it as a biomarker of the Parkinsonian state (Brown et al., 2001; Levy et al., 2002; Priori et al., 2004; Kühn et al., 2006; Wingeier et al., 2006; BronteStewart et al., 2009; Giannicola et al., 2010; Eusebio et al., 2011; Whitmer et al., 2012; Quinn et al., 2015). This resulted in the use of the resting state STN beta oscillopathy as a relevant neural input for adaptive DBS using a single threshold control policy, externalized leads, and a customized external neurostimulator in the acute, peri-operative state (Little et al., 2013). Consequently, the control policy that was embedded in the first generation of fully implanted bidirectional dBCIs (Activa ${ }^{\mathrm{TM}} \mathrm{PC}+\mathrm{S}-\mathrm{NexusE}$, Medtronic PLC, Minneapolis, MN, USA), was a single linear discriminator, corresponding to a single threshold of beta power. Using this we demonstrated that $60 \mathrm{~min}$ of closed-loop STN DBS was superior to clinical open-loop DBS for progressive bradykinesia (Figures 1B,C).

Bradykinesia $\left(\mathrm{V}_{\mathrm{rms}}\right)$ improved dramatically ON compared to OFF DBS (Figures 1A,B), and improved further on closed-loop DBS (Figure 1C). Progressive bradykinesia, or the waxingwaning and decreasing amplitude and speed of movement over time, was measured as the coefficient of variation of angular velocity $\left(\mathrm{CV}_{\text {vel }}\right) . \mathrm{CV}_{\text {vel }}$ was lower on closed-loop compared to open-loop DBS ( $12 \%$ compared to $24 \%$, respectively) demonstrating the superiority of closed-loop DBS. Both forms of DBS improved progressive bradykinesia compared to OFF DBS $\left(\mathrm{CV}_{\mathrm{vel}}=121 \%\right)$. There was a $63 \%$ reduction in the total electrical energy delivered during closed-loop DBS using a single active electrode compared to the optimized clinical DBS settings, which used a triple monopole.

\section{Decoding Neural Activity During Incremental Neuromodulation for Bradykinesia Led to the Development of the Dual-Threshold Control Policy Algorithm for Bidirectional dBCls}

Initially, it was difficult to discern between the effect of intensity and the effect of duration of STN DBS on the attenuation of beta 


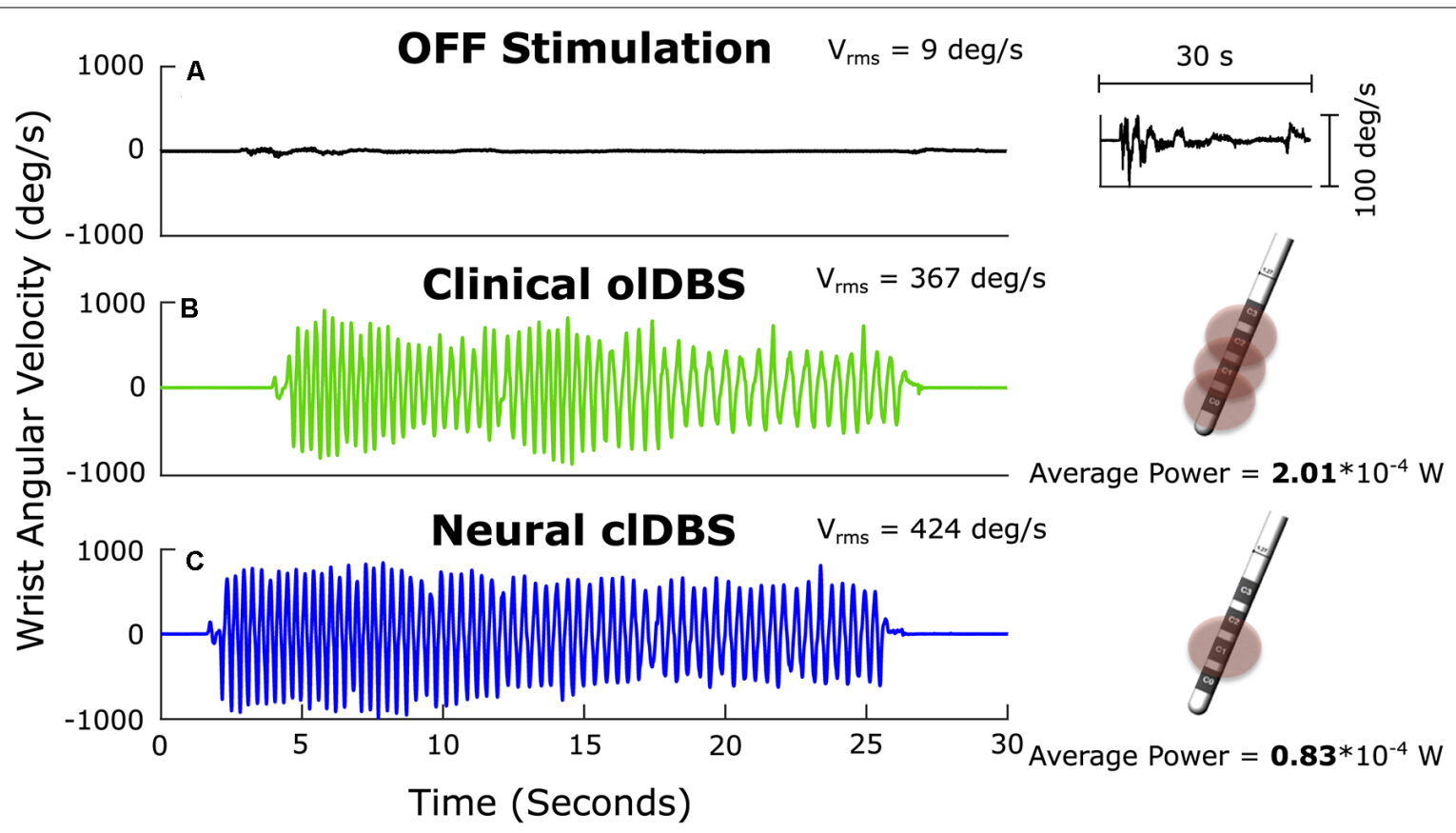

FIGURE 1 | Angular velocity traces measured during repetitive wrist flexion-extension OFF deep brain stimulation (DBS; A); the insert on the right at higher magnitude demonstrates the severe progressive bradykinesia, ON open-loop STN DBS (olDBS; B) and after 60 min of closed-loop STN DBS (cIDBS) (C). Schematic of the DBS lead demonstrates the use of a triple monopole during oIDBS and a single monopole during cIDBS. $V_{\text {rms: }}$ the root mean square angular velocity averaged over the trial.

band power, as there is a cumulative effect of longer periods of DBS on beta band power attenuation (Bronte-Stewart et al., 2009; Eusebio et al., 2011). We demonstrated that resting-state beta band power was attenuated in a dose-dependent manner during randomized epochs of different intensities of STN DBS (Whitmer et al., 2012).

A challenge for closed-loop DBS is to maintain a therapeutic effect while varying stimulation in real-time. Different control policies may have different goals and time scales. For instance, control policies that adjust DBS in real-time based on the appearance of symptoms [e.g., tremor or freezing of gait (FOG)] or based on rapid fluctuations of beta band power require a faster time scale (Malekmohammadi et al., 2016; Tinkhauser et al., 2017a; O’Day et al., 2020a; Petrucci et al., 2020a) compared to a control policy that adjusts DBS amplitude based on longer changes in beta band power due to fluctuating dopaminergic medication levels or the sleep-wake cycle (Arlotti et al., 2018; Velisar et al., 2019; Gilron et al., 2020). The control policies discussed in the next sections focused on time scales on the order of milliseconds. Initial single threshold control policies allowed for an "on-off" switch of neurostimulation based on the resting state beta power (Little et al., 2013); however, complete attenuation of STN beta power and/or decreasing DBS intensity toward completely off may not be optimal for motor performance (Blumenfeld et al., 2017). We developed a novel, customized, dual-threshold control policy based on the inverse relationship between DBS intensity and beta band power, and the direct relationship between DBS intensity and the improvement of bradykinesia off medication (Velisar et al., 2019). The effect of increasing DBS intensity on bradykinesia identified a minimum DBS intensity $\left(\mathrm{V}_{\min }\right)$ that resulted in the minimally acceptable improvement in bradykinesia in each individual. The beta power measured at $\mathrm{V}_{\min }$ was chosen as the upper beta power threshold. The lower beta threshold was the beta power halfway between that corresponding to $\mathrm{V}_{\min }$ and that corresponding to $\mathrm{V}_{\max }$. $\mathrm{V}_{\max }$ represented the DBS intensity above which adverse effects occurred for that individual. This resulted in a customized dual threshold policy that established a therapeutic window of DBS intensity where improvement in bradykinesia was acceptable. The policy instructed the $\mathrm{dBCI}$ to increase intensity when beta power exceeded the upper threshold, to stay constant when beta power remained between the dual thresholds and to decrease when beta power fell below the lower threshold. We implemented the customized dual threshold control policy and reported successful closed-loop STN DBS for bradykinesia and tremor in PD using a chronically implanted bidirectional dBCI [Activa $^{\mathrm{TM}} \mathrm{PC}+\mathrm{S}-\mathrm{NexusD3}$, Medtronic PLC, Minneapolis, MN, USA (Velisar et al., 2019)]. Closed-loop DBS resulted in $\sim 57 \%$ less total energy delivered compared to open loop DBS. We have recently demonstrated the superiority of closed loop STN DBS over clinical DBS for FOG in an individual; this experiment used beta power inputs and the customized dual threshold policy based on titrations of DBS intensity and measures of gait impairment and FOG (Petrucci et al., 2020b).

The temporal dynamics of beta band power (termed beta bursts) have been associated with clinical assessments of 
disease severity and with kinematic measures of bradykinesia, gait impairment, and FOG (Tinkhauser et al., 2017b; Anidi et al., 2018). There is a similar dose dependency between DBS intensity and beta burst duration both during rest and movement: increasing intensities of STN DBS were associated with shorter mean beta band durations and improved bradykinesia (Anderson et al., 2020). This dose-dependency of beta bursts suggests that a similar dual-threshold control policy in bidirectional DBCIs that monitors prolonged beta burst durations, as opposed to beta power specifically, could keep the DBS intensity within a therapeutic window.

\section{Optimization of Control Variables and Policies in Bidirectional dBCls in Freely Moving Activity States}

The ultimate goal of closed-loop DBS using a bidirectional dBCI is that neurostimulation will seamlessly adjust its parameters specific to the individual, their activity state, and their medication cycle (Arlotti et al., 2018). The ability to record synchronized neural and kinematic signals in freely moving individuals with $\mathrm{PD}$ using the implanted, sensing $\mathrm{dBCI}$ (Activa ${ }^{\mathrm{TM}} \mathrm{PC}+\mathrm{S}$, Medtronic PLC), led to the discovery of neural and kinematic signals that corresponded to abnormal movements such as bradykinesia, gait impairment, and FOG. These recordings have demonstrated that STN beta band power can be tracked during ongoing movement in $\mathrm{PD}$, that the peak frequency of the beta band did not change among rest, or finger, limb and axial movements, and that there was a subject-specific band of elevated beta power that was conserved throughout a variety of gait tasks (Blumenfeld et al., 2017; Anidi et al., 2018; Neuville et al., 2020). These contributions demonstrate that control policy algorithms in closed-loop DBS will be able to track, and do not need to adjust the frequency of, the beta band neural input in freely moving people with $\mathrm{PD}$.

Such synchronized recordings also revealed STN neural signatures that differentiated individuals with PD who exhibit FOG (freezers) from those who did not freeze (non-freezers) during non-freezing gait. Beta band power was lower, mean beta burst durations were longer, and there was greater beta Sample Entropy in freezers compared to non-freezers during non-freezing gait; freezers' gait was also more arrhythmic than that of non-freezers, even during "normal" walking (SyrkinNikolau et al., 2017; Anidi et al., 2018). In freezers, mean beta band burst durations were longer and alpha band $(8-12 \mathrm{~Hz})$ Sample Entropy was higher during periods of FOG, compared to during non-freezing gait. There was no difference in burst duration between the two groups in the resting state and burst duration was not correlated with mean power.

During open loop STN DBS at both $60 \mathrm{~Hz}$ and $140 \mathrm{~Hz}$, gait arrhythmicity and FOG improved and beta band power and burst durations decreased in freezers (Anidi et al., 2018; O’Day et al., 2020b). The normal gait rhythmicity and shorter burst durations were left unchanged during DBS in the non-freezers. This revealed a functional relevance of beta-band burst durations as neural inputs for closed-loop DBS for gait impairment and FOG using bidirectional dBCIs. Sixty Hertz DBS resulted in improved rhythmicity in both progressive limb bradykinesia and during forward-walking tasks (Blumenfeld et al., 2017; Anidi et al., 2018; O’Day et al., 2020b). A superior effect of $60 \mathrm{~Hz}$ to high-frequency DBS for FOG has been reported (Moreau et al., 2008; Xie et al., 2015), suggesting the need for control policies to include adjustments in neurostimulation intensity and frequency. A method of frequency-switching would allow a bidirectional $\mathrm{dBCI}$ to vary both intensity and frequency for optimal behavioral improvement and finer granularity of the effects of DBS.

\section{Using Relevant Behavioral Signals as Inputs to dBCls in Parkinson's Disease}

Kinematic signals specific to pathological episodic motor behaviors in PD, such as tremor and FOG, may be useful inputs to drive closed-loop neuromodulation. Resting tremor is a cardinal motor feature of PD and is an ideal behavioral input for closed-loop DBS: it is easily measured using a smartwatch, may be intermittent, and is different among individuals with PD, suggesting continuous neurostimulation may not be necessary. This was confirmed in the first behaviorally driven closed-loop DBS study, where resting tremor served as the input to the dBCI (Activa ${ }^{\mathrm{TM}} \mathrm{PC}+\mathrm{S}-\mathrm{NexusD}$ system, Medtronic PLC) and dual thresholds of tremor intensity defined the control policy (Malekmohammadi et al., 2016). Resting tremor was successfully attenuated and the time that the demand-based DBS system was activated varied from $11 \%$ to $99 \%$ (average of $51.5 \%$ ) of the time the continuous open-loop DBS was on. This highlights the possibility for more precise therapy for individuals with tremor-dominant $\mathrm{PD}$, who may benefit from a dBCI system that is not continuous. During resting tremors, underlying beta oscillations may be attenuated and neural inputs to dBCIs may not adequately control tremors (Shreve et al., 2017; Velisar et al., 2019). Enabling the capability for an additional or back up behavioral input may be an advantage for future bidirectional dBCIs.

\section{Neural and Kinematic Inputs Using Intensity- and Frequency-Based Control Policies to Provide Closed-Loop STN DBS for FOG in Parkinson's Disease}

The progress in the discovery of relevant control variables and policies for closed-loop DBS in PD fueled technological advances in the capabilities of bidirectional dBCIs. This has led us to the first series of investigations into the safety, feasibility, and efficacy of both neural and kinematic closed-loop STN DBS for FOG, using relevant neural and kinematic inputs and control policies that modulate either DBS intensity or frequency, using the investigative, next-generation bidirectional dBCI, the Summit ${ }^{\mathrm{TM}}$ $\mathrm{RC}+\mathrm{S}$ system (Medtronic PLC, Minneapolis, MN, USA; Figure 2; O’Day et al., 2020a; Petrucci et al., 2020a).

The Summit ${ }^{\mathrm{TM}} \mathrm{RC}+\mathrm{S}$ system can run both single and dual-threshold embedded algorithms. Similar to the Activa ${ }^{\mathrm{TM}}$ $\mathrm{PC}+\mathrm{S}-\mathrm{Nexus}-\mathrm{D} / \mathrm{E}$ systems, the Summit ${ }^{\mathrm{TM}} \mathrm{RC}+\mathrm{S}$ system has an Application Programming Interface (API) that allows for the development of distributed algorithms. We designed a 


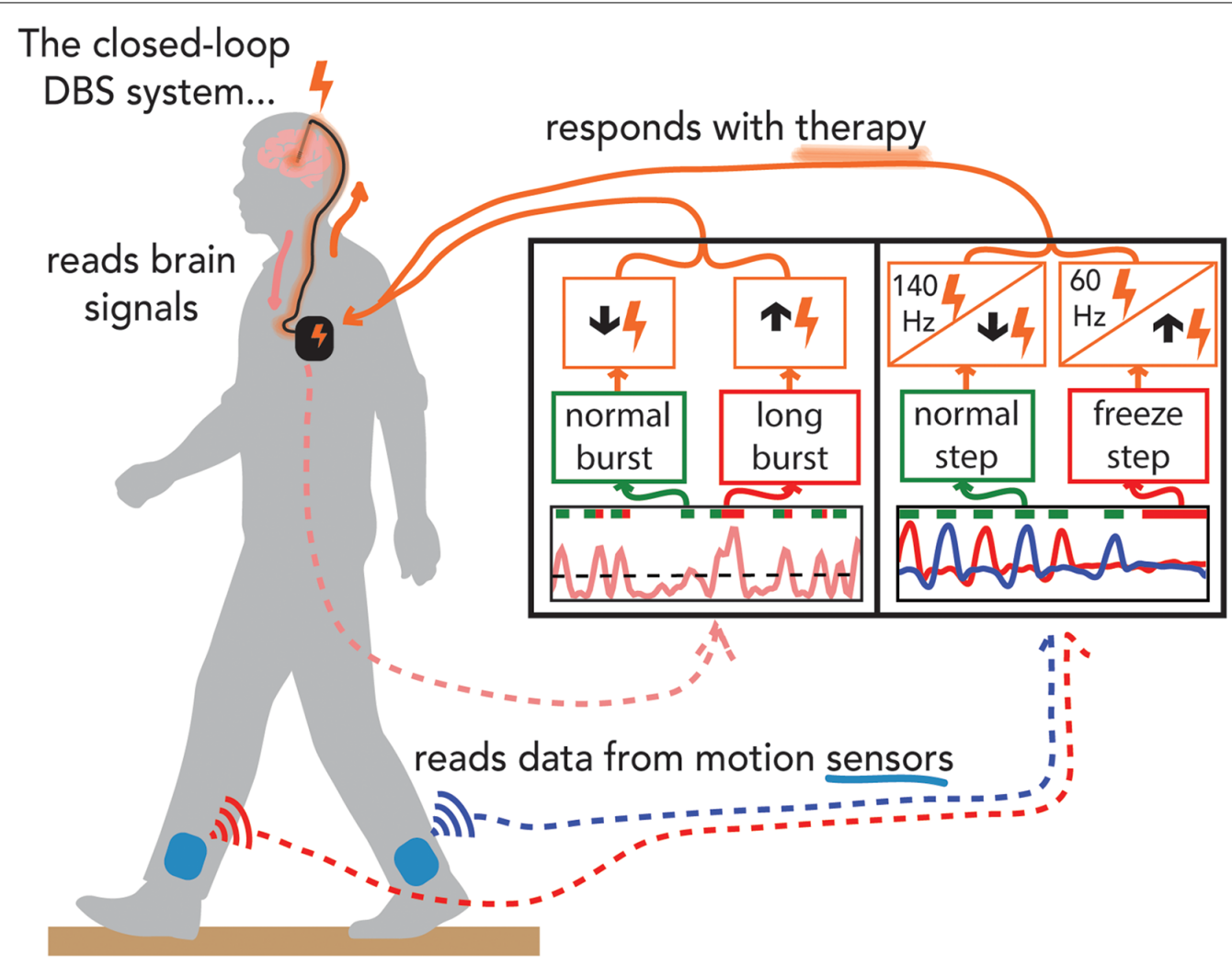

FIGURE 2 | Demonstration of experiments performed on the preclinical benchtop system using the Summit application programming interface. Schematic of the fully implanted bidirectional deep brain-computer interface $(\mathrm{dBCl})$ with data from the benchtop experiments. Left-hand panel: the neural input was beta band burst duration from the filtered local field potential; the single threshold control policy decided whether a neural burst was normal or long (pathological), and adapted closed-loop deep brain stimulation (CIDBS) by decreasing or increasing stimulation intensity respectively. Right-hand panel: the kinematic input was the shank angular velocity streamed from wearable inertial measurement units; a dual-threshold control policy was based on whether the step was determined to be normal, uncertain, or part of freezing of gait episode and adapted cIDBS by either (I) decreasing, not changing, or increasing stimulation intensity, OR, (II) by switching to $140 \mathrm{~Hz}$, staying unchanged or switching to $60 \mathrm{~Hz}$, respectively (right panel).

preclinical benchtop system using the accompanying Summit API (Medtronic Inc.) that allows for external control of the $\mathrm{RC}+\mathrm{S}$ neurostimulator using a PC-in-the-loop (Figure 2). The benchtop system played back previously recorded neural data and recorded output stimulation from the developed system. We used STN beta burst durations as the neural inputs and developed a novel faster time scale single threshold control policy algorithm that only increased stimulation intensity after the burst duration exceeded the inferred normal duration from simulated $1 / \mathrm{f}$ data, red bars in the left-hand panel in Figure 2 (Anderson et al., 2020). The benchtop system successfully adjusted stimulation in real-time in response to prolonged beta burst durations and demonstrated the feasibility of the algorithm by responding to pre-recorded STN data from an individual with PD (Petrucci et al., 2020a). We also successfully demonstrated for the first time the feasibility of kinematic closed-loop DBS for FOG using kinematic inputs relevant to impaired gait or FOG and policies that responded with adjustments of stimulation frequency or current intensity (Figure 2, right-hand panal) (O'Day et al., 2020a). This was done using real-time human subject kinematic data and kinematic data previously recorded from an individual with PD with gait impairment and FOG, allowing for real-time testing and iteration of these novel control policies using the test bench version of the Summit ${ }^{\mathrm{TM}} \mathrm{RC}+\mathrm{S}$ dBCI.

\section{CONCLUSION}

The ability to record neural signals from DBS leads implanted in deep brain structures has made it possible to deconstruct the neural code relevant to PD and establish that the STN beta oscillopathy is a robust and reliable input for closed-loop DBS using bidirectional deep Brain-Computer Interfaces (dBCIs) in freely moving people. This led to the first demonstration of the feasibility and efficacy of closed-loop DBS for progressive bradykinesia in PD, using beta band power as the input, a single threshold control policy, and a fully embedded bidirectional dBCI. Synchronized neural and kinematic recordings during incremental DBS intensities in freely moving individuals with PD resulted in novel customized dual-threshold control policy algorithms for closed-loop DBS, where DBS intensity fluctuated within a personalized safe and therapeutic window, driven by relevant beta band power or burst duration inputs. Beta driven closed-loop DBS using the dual-threshold algorithm and an implanted dBCI was demonstrated to be safe, feasible, 
and efficacious for bradykinesia, tremor, and FOG. The dual threshold algorithm was also used to demonstrate the efficacy of closed-loop DBS for tremor using tremor power as the input. Neural and kinematic characterization of gait impairment and FOG in PD and during $60 \mathrm{~Hz}$ and $140 \mathrm{~Hz}$ DBS has contributed to personalized neural and kinematic inputs, and frequency and intensity-based control policies for closed-loop STN DBS therapy for FOG in PD using next-generation bidirectional dBCIs.

The advances in discovery, innovation, and collaboration have led to the next generation of fully embedded investigative bidirectional dBCIs, in which both single and dual-threshold control policy algorithms are available (Percept ${ }^{\mathrm{TM}}$, Summit ${ }^{\mathrm{TM}}$ $\mathrm{RC}+\mathrm{S}$, Medtronic PLC, Minneapolis, MN, USA). Evolution in the understanding of relevant inputs and control policies in the first generation bidirectional $\mathrm{dBCIs}$ for $\mathrm{PD}$ and epilepsy has fueled similar discoveries for treatment of other neuropsychiatric disorders (Kundu et al., 2018; Rudebeck et al., 2019; Senova et al., 2019; Mankin and Fried, 2020). Advances in the understanding of the oscillopathies and circuitopathies of neuropsychiatric diseases are developing in parallel with advances in bidirectional dBCI technology. This is contributing to a paradigm shift in therapy, which will be more precise, customized to an individual's neural code, and will seamlessly adjust to their state of activity and medication cycle.

\section{DATA AVAILABILITY STATEMENT}

The raw data supporting the conclusions of this article will be made available by the authors, without undue reservation.

\section{REFERENCES}

Anderson, R. W., Kehnemouyi, Y. M., Neuville, R. S., Wilkins, K. B., Anidi, C. M., Petrucci, M. N., et al. (2020). A novel method for calculating beta band burst durations in Parkinson's disease using a physiological baseline. J. Neurosci. Methods 343:108811. doi: 10.1016/j.jneumeth.2020.108811

Anidi, C., O'Day, J. J., Anderson, R. W., Afzal, M. F., Syrkin-Nikolau, J., Velisar, A., et al. (2018). Neuromodulation targets pathological not physiological beta bursts during gait in Parkinson's disease. Neurobiol. Dis. 120, 107-117. doi: 10.1016/..nbd.2018.09.004

Arlotti, M., Marceglia, S., Foffani, G., Volkmann, J., Lozano, A. M., Moro, E., et al. (2018). Eight-hours adaptive deep brain stimulation in patients with Parkinson disease. Neurology 90, e971-e976. doi: 10.1212/WNL.00000000000 05121

Bergman, H., Wichmann, T., Karmon, B., and DeLong, M. R. (1994). The primate subthalamic nucleus. II. Neuronal activity in the MPTP model of parkinsonism. J. Neurophysiol. 72, 507-520. doi: 10.1152/jn.1994.72.2.507

Bevan, M. D., Magill, P. J., Terman, D., Bolam, J. P., and Wilson, C. J. (2002). Move to the rhythm: oscillations in the subthalamic nucleus-external globus pallidus network. Trends Neurosci. 25, 525-531. doi: 10.1016/S0166-2236(02) 02235-X

Blumenfeld, Z., Koop, M. M., Prieto, T. E., Shreve, L. A., Velisar, A., Quinn, E. J., et al. (2017). Sixty-hertz stimulation improves bradykinesia and amplifies subthalamic low-frequency oscillations. Mov. Disord. 32, 80-88. doi: $10.1002 / \mathrm{mds} .26837$

Bronte-Stewart, H., Barberini, C., Koop, M. M., Hill, B. C., Henderson, J. M., and Wingeier, B. (2009). The STN beta-band profile in Parkinson's disease is

\section{ETHICS STATEMENT}

The studies involving human participants were reviewed and approved by the Stanford University Institutional Review Board. The patients/participants provided their written informed consent to participate in this study.

\section{AUTHOR CONTRIBUTIONS}

HB-S, MP, JO'D, JP, YK, KW, GO and SH contributed to the conceptualization of the work and the writing and editing of the manuscript. MP, JO'D, MA, and KW contributed to the visualization of the results. MA contributed to software and data curation, as well as formal analysis.

\section{FUNDING}

This work was supported by the Michael J. Fox Foundation (9605), National Institute of Neurological Disorders and Stroke (NINDS) Grant 5R21 NS096398-02, Robert and Ruth Halperin Foundation, John A. Blume Foundation, Helen M. Cahill Award for Research in Parkinson's Disease, NINDS UH3NS107709, the Stanford Bio-X Graduate Fellowship, and Medtronic PLC, who provided the devices used in this study but no additional financial support.

\section{ACKNOWLEDGMENTS}

We would like to thank Jeffrey Herron, Jaimie Henderson, and previous members of the lab for their contribution to this body of work throughout the years.

stationary and shows prolonged attenuation after deep brain stimulation. Exp. Neurol. 215, 20-28. doi: 10.1016/j.expneurol.2008.09.008

Brown, P. (2003). Oscillatory nature of human basal ganglia activity: relationship to the pathophysiology of parkinson's disease. Mov. Disord. 18, 357-363. doi: $10.1002 / \mathrm{mds} .10358$

Brown, P., Oliviero, A., Mazzone, P., Insola, A., Tonali, P., and Di Lazzaro, V. (2001). Dopamine dependency of oscillations between subthalamic nucleus and pallidum in Parkinson's disease. J. Neurosci. 21, 1033-1038. doi: 10.1523/jneurosci.21-03-01033.2001

de Solages, C., Hill, B. C., Koop, M. M., Henderson, J. M., and Bronte-Stewart, H. (2010). Bilateral symmetry and coherence of subthalamic nuclei beta band activity in Parkinson's disease. Exp. Neurol. 221, 260-266. doi: 10.1016/j. expneurol.2009.11.012

Deuschl, G., Schade-Brittinger, C., Krack, P., Volkmann, J., Schäfer, H., Bötzel, K. et al. (2006). A randomized trial of deep-brain stimulation for Parkinson's disease. N. Engl. J. Med. 355, 896-908. doi: 10.1056/NEJMoa060281

Dorval, A. D., Muralidharan, A., Jensen, A. L., Baker, K. B., and Vitek, J. L. (2015). Information in pallidal neurons increases with parkinsonian severity. Parkinsonism Relat. Disord. 21, 1355-1361. doi: 10.1016/j.parkreldis.2015. 09.045

Edwards, C. A., Kouzani, A., Lee, K. H., and Ross, E. K. (2017). Neurostimulation Devices for the treatment of neurologic disorders. Mayo Clin. Proc. 92, 1427-1444. doi: 10.1016/j.mayocp.2017.05.005

Eusebio, A., Thevathasan, W., Doyle Gaynor, L., Pogosyan, A., Bye, E., Foltynie, T., et al. (2011). Deep brain stimulation can suppress pathological synchronisation in parkinsonian patients. J. Neurol. Neurosurg. Psychiatry 82, 569-573. doi: 10.1136/jnnp.2010.217489 
Feingold, J., Gibson, D. J., Depasquale, B., and Graybiel, A. M. (2015). Bursts of beta oscillation differentiate postperformance activity in the striatum and motor cortex of monkeys performing movement tasks. Proc. Natl. Acad. Sci. U S A 112, 13687-13692. doi: 10.1073/pnas.1517629112

Fetz, E. E. (2015). Restoring motor function with bidirectional neural interfaces. Prog. Brain Res. 218, 241-252. doi: 10.1016/bs.pbr.2015.01.001

Giannicola, G., Marceglia, S., Rossi, L., Mrakic-Sposta, S., Rampini, P., Tamma, F., et al. (2010). The effects of levodopa and ongoing deep brain stimulation on subthalamic beta oscillations in Parkinson's disease. Exp. Neurol. 226, 120-127. doi: 10.1016/j.expneurol.2010.08.011

Gilron, R., Little, S., Perrone, R., Wilt, R., De Hemptinne, C., Maria, S., et al (2020). Chronic wireless streaming of invasive neural recordings at home for circuit discovery and adaptive stimulation. BioRxiv [Preprint]. doi: 10.1101/2020.02. 13.948349

He, B. J. (2014). Scale-free brain activity: past, present and future. Trends Cogn. Sci. 18, 480-487. doi: 10.1016/j.tics.2014.04.003

Krack, P., Batir, A., Van Blercom, N., Chabardes, S., Fraix, V., Ardouin, C., et al. (2003). Five-year follow-up of bilateral stimulation of the subthalamic nucleus in advanced Parkinson's disease. N. Engl. J. Med. 349, 1925-1934. doi: 10.1056/NEJMoa035275

Kühn, A. A., Kupsch, A., Schneider, G. H., and Brown, P. (2006). Reduction in subthalamic $8-35 \mathrm{~Hz}$ oscillatory activity correlates with clinical improvement in Parkinson's disease. Eur. J. Neurosci. 23, 1956-1960. doi: 10.1111/j.14609568.2006.04717.x

Kundu, B., Brock, A. A., Englot, D. J., Butson, C. R., and Rolston, J. D. (2018). Deep brain stimulation for the treatment of disorders of consciousness and cognition in traumatic brain injury patients: a review. Neurosurg. Focus 45:E14. doi: 10.3171/2018.5.FOCUS18168

Levy, R., Ashby, P., Hutchison, W. D., Lang, A. E., Lozano, A. M., and Dostrovsky, J. O. (2002). Dependence of subthalamic nucleus oscillations on movement and dopamine in Parkinson's disease. Brain 125, 1196-1209. doi: 10.1093/brain/awf128

Little, S., Pogosyan, A., Neal, S., Zavala, B., Zrinzo, L., Hariz, M., et al. (2013). Adaptive deep brain stimulation in advanced Parkinson disease. Ann. Neurol. 74, 449-457. doi: 10.1002/ana.23951

Malekmohammadi, M., Herron, J., Velisar, A., Blumenfeld, Z., Trager, M. H., Chizeck, H. J., et al. (2016). Kinematic adaptive deep brain stimulation for resting tremor in Parkinson's disease. Mov. Disord. 31, 426-428. doi: $10.1002 / \mathrm{mds} .26482$

Mallet, N., Pogosyan, A., Márton, L. F., Bolam, J. P., Brown, P., and Magill, P. J. (2008). Parkinsonian beta oscillations in the external globus pallidus and their relationship with subthalamic nucleus activity. J. Neurosci. 28, 14245-14258. doi: 10.1523/JNEUROSCI.4199-08.2008

Mankin, E. A., and Fried, I. (2020). Modulation of human memory by deep brain stimulation of the entorhinal-hippocampal circuitry. Neuron 106, 218-235. doi: 10.1016/j.neuron.2020.02.024

Moreau, C., Defebvre, L., Destée, A., Bleuse, S., Clement, F., Blatt, J. L., et al. (2008). STN-DBS frequency effects on freezing of gait in advanced Parkinson disease. Neurology 71, 80-84. doi: 10.1212/01.wnl.0000303972. 16279.46

Muralidharan, A., Jensen, A. L., Connolly, A., Hendrix, C. M., Johnson, M. D., Baker, K. B., et al. (2016). Physiological changes in the pallidum in a progressive model of Parkinson's disease: are oscillations enough? Exp. Neurol. 279, 187-196. doi: 10.1016/j.expneurol.2016.03.002

Mussa-Ivaldi, F. A., Alford, S. T., Chiappalone, M., Fadiga, L., Karniel, A., Kositsky, M., et al. (2010). New perspectives on the dialogue between brains and machines. Front. Neurosci. 4:44. doi: 10.3389/neuro.01. 008.2010

Neuville, R. S., Anderson, R. W., Petrucci, M. N., Parker, J. E., Wilkins, K. B., Velisar, A., et al. (2020). Pathological beta burst dynamics are conserved across different movements in Parkinson's disease. MedRxiv [Preprint]. doi: 10.1101/2020.04.12.20063032

Nini, A., Feingold, A., Slovin, H., and Bergman, H. (1995). Neurons in the globus pallidus do not show correlated activity in the normal monkey, but phase-locked oscillations appear in the MPTP model of Parkinsonism. J. Neurophysiol. 74, 1800-1805. doi: 10.1152/jn.1995.74.4.1800

O’Day, J., Kehnemouyi, Y., Petrucci, M., Anderson, R., Herron, J., and BronteStewart, H. (2020a). "Demonstration of kinematic-based closed-loop deep brain stimulation for mitigating freezing of gait in people with Parkinson's disease," in 42nd Annual International Conferences of the IEEE Engineering in Medicine and Biology Society, Montreal, QC, 3612-3616.

O’Day, J., Syrkin-Nikolau, J., Anidi, C., Kidzinski, L., Delp, S., and BronteStewart, H. (2020b). The turning and barrier course reveals gait parameters for detecting freezing of gait and measuring the efficacy of deep brain stimulation. PLoS One 15:e0231984. doi: 10.1371/journal.pone.0231984

Petrucci, M., Anderson, R., O'day, J., Kehnemouyi, Y., and Herron, J. (2020a). “A closed-loop deep brain stimulation approach for mitigating burst durations in people with Parkinson's disease," in 42nd Annual International Conferences of the IEEE Engineering in Medicine and Biology Society, Montreal, QC, $3617-3620$.

Petrucci, M., Neuville, R., Afzal, M., Velisar, A., Anidi, C., Anderson, R., et al. (2020b). Neural closed loop deep brain stimulation for freezing of gait. Brain Stimul. 13, 1320-1322. doi: 10.1016/j.brs.2020.06.018

Priori, A., Foffani, G., Pesenti, A., Tamma, F., Bianchi, A. M., Pellegrini, M., et al. (2004). Rhythm-specific pharmacological modulation of subthalamic activity in Parkinson's disease. Exp. Neurol. 189, 369-379. doi: 10.1016/j.expneurol. 2004.06.001

Priori, A., Foffani, G., Rossi, L., and Marceglia, S. (2013). Adaptive deep brain stimulation (aDBS) controlled by local field potential oscillations. Exp. Neurol. 245, 77-86. doi: 10.1016/j.expneurol.2012.09.013

Quinn, E. J., Blumenfeld, Z., Velisar, A., Koop, M. M., Shreve, L. A., Trager, M. H., et al. (2015). Beta oscillations in freely moving Parkinson's subjects are attenuated during deep brain stimulation. Mov. Disord. 30, 1750-1758. doi: $10.1002 / \mathrm{mds} .26376$

Rudebeck, P. H., Rich, E. L., and Mayberg, H. S. (2019). From bed to bench side: reverse translation to optimize neuromodulation for mood disorders. Proc. Natl. Acad. Sci. U S A 116, 26288-26296. doi: 10.1073/pnas.19022 87116

Schnitzler, A., and Gross, J. (2005). Normal and pathological oscillatory communication in the brain. Nat. Rev. Neurosci. 6, 285-296. doi: 10.1038/nrn1650

Schuepbach, W. M. M., Rau, J., Knudsen, K., Volkmann, J., Krack, P., Timmermann, L., et al. (2013). Neurostimulation for Parkinson's disease with early motor complications. N. Engl. J. Med. 368, 610-622. doi: 10.1056/NEJMoa1205158

Senova, S., Clair, A. H., Palfi, S., Yelnik, J., Domenech, P., and Mallet, L. (2019). Deep brain stimulation for refractory obsessive-compulsive disorder: towards an individualized approach. Front. Psychiatry 10:905. doi: 10.3389/fpsyt.2019. 00905

Shreve, L. A., Velisar, A., Malekmohammadi, M., Koop, M. M., Trager, M., Quinn, E. J., et al. (2017). Subthalamic oscillations and phase amplitude coupling are greater in the more affected hemisphere in Parkinson's disease. Clin. Neurophysiol. 128, 128-137. doi: 10.1016/j.clinph.2016. 10.095

Syrkin-Nikolau, J., Koop, M. M., Prieto, T., Anidi, C., Afzal, M. F., Velisar, A., et al. (2017). Subthalamic neural entropy is a feature of freezing of gait in freely moving people with Parkinson's disease. Neurobiol. Dis. 108, 288-297. doi: 10.1016/j.nbd.2017.09.002

Tinkhauser, G., Pogosyan, A., Little, S., Beudel, M., Herz, D. M., Tan, H., et al. (2017a). The modulatory effect of adaptive deep brain stimulation on beta bursts in Parkinson's disease. Brain 140, 1053-1067. doi: 10.1093/brain/ awx010

Tinkhauser, G., Pogosyan, A., Tan, H., Herz, D. M., Kühn, A. A., and Brown, P. (2017b). Beta burst dynamics in Parkinson's disease OFF and ON dopaminergic medication. Brain 140, 2968-2981. doi: 10.1093/brain/ awx 252

Trager, M. H., Koop, M. M., Velisar, A., Blumenfeld, Z., Nikolau, J. S., Quinn, E. J., et al. (2016). Subthalamic beta oscillations are attenuated after withdrawal of chronic high frequency neurostimulation in Parkinson's disease. Neurobiol. Dis. 96, 22-30. doi: 10.1016/j.nbd.2016.08.003

Velisar, A., Syrkin-Nikolau, J., Blumenfeld, Z., Trager, M. H., Afzal, M. F., Prabhakar, V., et al. (2019). Dual threshold neural closed loop deep brain stimulation in Parkinson disease patients. Brain Stimul. 12, 868-876. doi: 10.1016/j.brs.2019.02.020

Wang, J., Johnson, L. A., Jensen, A. L., Baker, K. B., Molnar, G. F., Johnson, M. D., et al. (2017). Network-wide oscillations in the parkinsonian state: alterations 
in neuronal activities occur in the premotor cortex in parkinsonian nonhuman primates. J. Neurophysiol. 117, 2242-2249. doi: 10.1152/jn.000 11.2017

Weaver, F., Follett, K., Hur, K., Ippolito, D., and Stern, M. (2005). Deep brain stimulation in Parkinson disease: a meta-analysis of patient outcomes. J. Neurosurg. 103, 956-967. doi: 10.3171/jns.2005.103. 6.0956

Whitmer, D., de Solages, C., Hill, B., Yu, H., Henderson, J. M., and BronteStewart, H. (2012). High frequency deep brain stimulation attenuates subthalamic and cortical rhythms in Parkinson's disease. Front. Hum. Neurosci. 6:155. doi: 10.3389/fnhum.2012.00155

Wichmann, T., Bergman, H., and DeLong, M. R. (1994). The primate subthalamic nucleus. I. Functional properties in intact animals. J. Neurophysiol. 72, 494-506. doi: 10.1152/jn.1994.72.2.494

Williams, A., Gill, S., Varma, T., Jenkinson, C., Quinn, N., Mitchell, R., et al. (2010). Deep brain stimulation plus best medical therapy versus best medical therapy alone for advanced Parkinson's disease (PD SURG trial): a randomised, open-label trial. Lancet Neurol. 9, 581-591. doi: 10.1016/S1474-4422(10) 70093-4

Wingeier, B., Tcheng, T., Koop, M. M., Hill, B. C., Heit, G., and BronteStewart, H. M. (2006). Intra-operative STN DBS attenuates the prominent beta rhythm in the STN in Parkinson's disease. Exp. Neurol. 197, 244-251. doi: 10.1016/j.expneurol.2005.09.016

Xie, T., Vigil, J., MacCracken, E., Gasparaitis, A., Young, J., Kang, W., et al. (2015). Low-frequency stimulation of STN-DBS reduces aspiration and freezing of gait in patients with PD. Neurology 84, 415-420. doi: 10.1212/WNL. 0000000000001184

Conflict of Interest: Dr. HB-S serves on the Scientific Advisory Board for Medtronic PLC.

The remaining authors declare that the research was conducted in the absence of any commercial or financial relationships that could be construed as a potential conflict of interest.

Copyright ( 2020 Bronte-Stewart, Petrucci, O’Day, Afzal, Parker, Kehnemouyi, Wilkins, Orthlieb and Hoffman. This is an open-access article distributed under the terms of the Creative Commons Attribution License (CC BY). The use, distribution or reproduction in other forums is permitted, provided the original author(s) and the copyright owner(s) are credited and that the original publication in this journal is cited, in accordance with accepted academic practice. No use, distribution or reproduction is permitted which does not comply with these terms. 